\title{
Depression in cancer patients: Pathogenesis, implications and treatment (Review)
}

\author{
HAMISH R. SMITH \\ School of Medicine and Dentistry, James Cook University, Townsville, Queensland 4811, Australia
}

Received June 25, 2014; Accepted February 2, 2015

DOI: 10.3892/ol.2015.2944

\begin{abstract}
Depression is a common comorbidity in cancer cases, affecting $>10 \%$ of patients. A cancer diagnosis is life-changing, and is a source of considerable psychological and emotional stress. Non-pathological sadness may be a normal response to a cancer diagnosis, however, stress beyond the coping mechanisms of patients may result in major depressive disorder. The current review, in addition to the obvious psychosocial elements of depression, explores its biological mechanisms, including tissue damage, inflammatory mediators and the chronic stress response, and how these immune and endocrine pathways may underlie depression in cancer. Possible iatrogenic causes of depression in cancer are also explored. There is a strong need to identify and treat depression in cancer patients in order to increase quality of life and reduce mortality. The most popular clinical and potential future biochemical screening tools for depression in cancer are briefly discussed. The interventions used will vary for every patient, but may include psychosocial therapies or pharmacotherapy; however, a paucity of research on the most effective management of depression in cancer means the optimal combination of therapies is unknown. Selection of antidepressants should be carefully considered, given the common side effects of chemotherapy (such as nausea), and the necessity to avoid serious interactions, including reducing the effectiveness of chemotherapeutic drugs. The possible link between the chronic stress response, which may predispose patients to depression, and the risk of mortality from cancer is also explored. The complex interactions between the endocrine, nervous and immune systems, which continue to be elucidated, may offer the opportunity for the development of more rapid and efficacious treatments for depression in cancer in the future.
\end{abstract}

Correspondence to: Mr. Hamish Ronald Smith, School of Medicine and Dentistry, James Cook University, 39 James Cook Drive, Townsville, QLD 4811, Australia

E-mail: hamish.smith@my.jcu.edu.au

Key words: depression, psycho-oncology, psychoneuroimmunology, psychosomatics

\section{Contents}

1. Introduction

2. Symptoms and diagnosis

3. Pathogenesis

4. Implications

5. Management

6. Conclusion

\section{Introduction}

Cancer is a life-threatening and feared diagnosis, and is a source of great distress in patients. A cancer diagnosis generates a higher sense of distress than non-neoplastic diseases with poorer prognoses (1). High levels of mental distress for sustained periods of time in cancer patients may lead to anxiety, depression or both (2). This mixed symptomatology is very common, with two thirds of cancer patients with depression also expressing clinically significant levels of anxiety (3).

Depression leads to a poorer quality of life (QOL) and compromises patient outcomes, with depression resulting in higher rates of mortality in cancer $(4,5)$. A meta-analysis revealed that minor or major depression increases mortality rates by up to $39 \%$, and that patients displaying even few depressive symptoms may be at a $25 \%$ increased risk of mortality (6). The impact of mood and mental wellbeing on cancer progression is considered important by doctors and patients, with $>70 \%$ of oncologists and $85 \%$ of patients believing that mood affects the progression of cancer (7).

The rate of depression in cancer patients is thought to be up to three times higher than in the general population (2). Studies using Diagnostic for Statistical Manual of Mental Disorders (DSM) criteria (8) for major depressive disorder (MDD) have identified a variety of prevalences ranging from $2.0-43.5 \%(9,10)$, whilst palliative care wards have documented rates of depression as high as $49.0 \%$ (11). The wide range of reported prevalences may be due to differences in assessment tools, variation in the types of patients interviewed, varying age groups, varying gender proportions, inpatient status and other factors. A study by Linden et al (2), and a comprehensive literature review by $\mathrm{Ng}$ et al (12) detailing rates of depression in $>9,000$ patients, each in a variety of settings and ages, calculated the prevalence as $10.8 \%$ and $12.9 \%$, respectively. In addition, a further $16 \%$ of patients are reported to have subclinical, yet still damaging, depression $(2,5)$. 
The site of primary cancer also influences rates of depression, with depression being most common in pancreatic and lung cancers and lowest in invasive skin cancer (2). Age also influences prevalence; evidence suggests that children and adolescents with cancer are no more depressed than healthy controls $(13,14)$, whilst for some cancers among adults, age was inversely associated with depression (2). Gender is also a significant factor: In some cancer types female patients were found to be two to three times more likely to experience depression than males (2). Levels of psychological stress and depression also vary over the course of the disease and are highest around the time of diagnosis (2). However, rates of depression in cancer survivors five years following diagnosis were demonstrated to be comparable to the broader public, at $4 \%$ (15).

Metastases and cancer pain have also been associated with higher levels of depression (11). The prevalence of depression in patients with high levels of pain compared with low pain levels is significantly higher; one study observed that depression occurred in $33 \%$ of those in high amounts of pain, compared with $13 \%$ in those with low levels of pain, suggesting that pain may be a causative factor in depression (16).

\section{Symptoms and diagnosis}

For the diagnosis of MDD according to the DSM, the patient must have either a depressed mood or a diminished level of interest or pleasure in activities for at least two weeks. In addition, four or more of the following must be present: Significant weight change, hypersomnia or insomnia, psychomotor changes, fatigue, feelings of guilt or worthlessness, poor concentration or cognition and recurrent thoughts of death or suicide. The symptoms must also cause significant distress or impairment, and must not be a result of the physiological effects of a substance or disease (8).

The somatic presentations of depression, including fatigue, loss of appetite, weight change and poor cognition, can be dismissed by clinicians simply as symptoms of cancer or side effects of treatment, which leads to decreased detection of the disorder. In one study, appetite changes and reduced cognitive ability were found to be positively associated with anhedonia, whereas fatigue and sleep difficulties were not, even after adjusting for cancer pain and physical functioning. This suggests that reduced appetite and poor cognition may be more useful symptoms in diagnosing depression in cancer (17). Feelings of guilt and failure are also lower among depressed cancer patients, at $4 \%$, compared with depressed but otherwise healthy patients, at $56.5 \%$ (18). Diagnosis is complex as it may be normal for some of the above symptoms to be present given the immense physical and psychological strain on patients. Patients may also battle with other behavioural changes, including sleep difficulties, poor cognition, anorexia, social withdrawal and fatigue $(19,20)$.

Screening. Screening is important to identify patients who are depressed and would benefit from further support $(4,5)$. The Hospital Anxiety and Depression Scale - Depression (HADS-D) (21) is a self-administered screening questionnaire with seven questions regarding depressive symptoms, each scored by the patient from $0-3$. Scores of $\geq 8$ are indicative of probable depression, whilst scores of $\geq 11$ are highly indicative of depression (21).

A study measuring interleukin-6 (IL-6) levels and relative diurnal cortisol variation in depressed cancer patients revealed that IL-6 levels are increased by a factor of seven $(18.7 \mathrm{pg} / \mathrm{ml}$ vs. $2.7 \mathrm{pg} / \mathrm{ml})$, whilst relative diurnal cortisol variation is decreased by a factor of six (11.7\% vs. $60.6 \%)$ among cancer patients with depression compared with those without depression. Screening tests for depression in cancer patients using diurnal cortisol variation, at a cut-off value of $35 \%$, provided the highest specificity and sensitivity, at $88 \%$ and $81 \%$, respectively; these tests may therefore be useful in detecting depression in cancer patients (22).

\section{Pathogenesis}

Depression in cancer is a multifactorial disorder involving psychosocial, biological and even iatrogenic causes.

Psychological and social. Depression exists on a continuum ranging from non-pathological sadness, to an adjustment disorder, to subclinical depression to major depression. Depression may result from stress that overwhelms a person's ability to adjust to changes in life, leading to a persistently low mood, despair, anhedonia and feelings of hopelessness. Emotional stress may stem from a bleak prognosis or the extreme uncertainty that patients endure. This is compounded by the negative effects that a cancer diagnosis and treatment may have on a patient's job, family, physical appearance, abilities, independence and finances. Those with maladaptive coping strategies, previous mental illness and poor communication with medical practitioners are at particularly high risk of developing depression (10). Strong emotional support from friends and family and an optimistic view are protective factors from developing depression (2).

Inflammation. Evidence suggests that, in addition to the obvious emotional and psychosocial dimensions of depression in cancer, biological mechanisms may also be important. Tissue destruction by surgery, chemotherapy or radiotherapy leads to damage-associated molecular patterns (DAMPs) on damaged tissue, which bind to pattern recognition receptors (PRRs) on leukocytes, particularly macrophages, causing the expression of the transcription factor nuclear factor- $\kappa-\beta(\mathrm{NF} \kappa \beta)$ and the production of numerous pro-inflammatory cytokines, including interleukin-1 (IL-1), interferon- $\alpha$ (INF- $\alpha$ ), IL-6 and tumour necrosis factor- $\alpha$ (TNF- $\alpha$ ) (23). Ionising radiation and some chemotherapeutic agents are also able to directly stimulate $\mathrm{NF \kappa} \beta$ independently of tissue damage and further increase inflammatory mediator expression (24). Psychosocial stress in healthy patients has also been shown to induce $\mathrm{NF} \kappa \beta$ expression (25).

TNF- $\alpha$, IL-1 and other cytokines have also been observed to increase the activity and expression of serotonin (5-hydroxytryptamine; 5-HT) and noradrenaline (NA) reuptake transporters, through activation of the p38 mitogen activated protein kinase (MAPK). This effectively lowers synaptic concentrations of 5-HT and NA, and can lead to depressive behaviours (26-28). Proinflammatory cytokines also increase the secretion of corticotropin releasing hormone (CRH). $\mathrm{CRH}$ 
itself can cause behavioural changes including those observed in depression (29). Furthermore, these cytokines reduce levels of neural growth factors, such as brain-derived neurotrophic factor (BDNF), which is key for neurogenesis. Low levels of BDNF and neurogenesis have been implicated in the pathogenesis of depression (30).

In non-human primate studies, INF- $\alpha$ has been observed to decrease the expression of dopamine (DA)-2 receptors and reduce striatal DA release, leading to feelings of anhedonia. Although the mechanisms behind this and any possible pharmacological strategies remain to be elucidated (31), INF- $\alpha$ also results in decreased conversion of phenylalanine to tyrosine, which causes reduced downstream formation of DA in the brain and, potentially, depressive symptoms (32).

Pro-inflammatory cytokines, particularly TNF- $\alpha$, have been shown to increase the activity of an enzyme called indolamine-2,3-dioxygenase (IDO), which degrades tryptophan. Tryptophan is the chemical precursor to 5-HT; therefore its destruction results in reduced 5-HT levels (33). It is well established that tryptophan levels are decreased relative to monoamine levels in the brains of depressed patients (34). Induction of IDO also causes the formation of neurodegenerative tryptophan catabolites (TRYCATs). These TRYCATs, including kynurenine and quinolinic acid, may also be important in depression in cancer, as they are proinflammatory and neurotoxic in the brain. Quinolinic acid is a potent $\mathrm{N}$-methyl-D-aspartate (NMDA) receptor agonist and is able to induce lipid peroxidation in neurons by excitotoxicity, whilst kynurenine has been demonstrated to be anxiogenic (35). In severe depression, levels of quinolinic acid have been observed to be raised in several regions throughout the anterior cingulate cortex. Neurotoxicity and degeneration, particularly of the hippocampus, have been implicated in the development of depression (36).

Chronic stress. Living with the physical and psychological burden of a cancer diagnosis and treatment is a form of chronic stress. During stress, the sympathetic nervous system (SNS) is stimulated, the parasympathetic nervous system (PSNS) is inhibited and the hypothalamic-pituitary-adrenal (HPA) axis is activated (26,27,37-39). The stimulation of the HPA-axis should lead to the downstream release of endogenous glucocorticoids, potent anti-inflammatories which normally inhibit pro-inflammatory cytokine production. However, PRR activation by DAMPs causes the intracellular glucocorticoid receptor (GCR) in leukocytes to be inactivated by p38 MAPK; the anti-inflammatory effect of glucocorticoids is therefore attenuated, and cytokine expression is increased $(23,40)$. Proinflammatory cytokines may also lead to decreased sensitivity in the remaining activated GCRs, resulting in a further decrease in anti-inflammatory activity of glucocorticoids and increased cytokine production (41). Additionally, the increased NA, released due to chronic SNS stimulation, binds to adrenergic receptors on macrophages, which causes further NFk $\beta$ activation and cytokine expression, leading to further reductions in 5-HT and NA concentrations $(27,28)$. Usually PSNS activity should inhibit the production of pro-inflammatory cytokines; acetylcholine binds to nicotinic receptors on leukocytes and inhibits the production of $\mathrm{NF} \kappa \beta$ (38). However, the PSNS is inhibited in a chronic stress state, instead leading to increased cytokine formation, which acts to further alter neurotransmitter levels $(38,39)$.

Medications. Some medications have been implicated in causing depression-like symptoms in cancer patients. The DA receptor-2 antagonist haloperidol, occasionally used in the treatment of chemotherapy-associated nausea, reduces dopaminergic transmission in the brain and has been linked to the development of depressive symptoms (42). Immunotherapy agents, including INF- $\alpha$, used in some cancers have been reported to cause depression in up to $50 \%$ of patients by the mechanisms explained above (26-28).

\section{Implications}

Chemotherapy and the health care system. In a cohort study investigating breast cancer outcomes in patients with depression, only $51 \%$ of the study group (breast cancer patients with depression) accepted and commenced chemotherapy, compared with $92 \%$ of the control group (breast cancer patients without depression) (4). Depression affects treatment participation, causes poorer outcomes and results in higher mortality $(4,5)$.

Depression causes a greatly diminished QOL in cancer patients by worsening physical symptoms, and increases the negative impact on patients and their families throughout the course of the disease $(2,43)$. For example, during treatment for breast cancer, patients with higher pre-chemotherapy levels of fatigue, depression and sleep disturbances suffered increased levels of these symptoms during treatment, which had severe and adverse effects on their QOL (44). Depression in cancer patients increases the length of hospital stay and resource use, increasing health expenditure (45). Depressed cancer patients are also at a higher risk of suicide compared with the general public (46).

Cancer progression and depression. Depressed cancer patients are at increased risk of mortality compared with non-depressed patients (5). Although treatment non-participation accounts for some of the increased risk, evidence from animal studies and human studies suggests that the chronic stress response, which may contribute to the development of depression in cancer, may also contribute to increased cancer invasiveness, reduced tumour surveillance by the body, increased angiogenesis, reduced tumour suppressor gene activity and reduced cellular apoptosis $(6,47-51)$.

Immune modulation. Numerous immune mechanisms are thought to underlie the increased risk of mortality of depressed patients compared with non-depressed patients (6). Depression in otherwise healthy adults reduces the number of circulating natural killer (NK) cells, which normally have a tumour surveillance role, and the same is predicted to apply in cancer patients (47). In ovarian cancer patients, depressed and anxious moods were associated with significantly impaired $\mathrm{T}$ helper 1 cell levels, cytotoxic $\mathrm{T}$ lymphocyte levels and interferon- $\gamma$ (INF- $\gamma$ ) secretion in both the microenvironment of the tumour and peripheral blood (48). In one murine study, which involved exposing stressed and unstressed groups of ultraviolet radiation-sensitive mice (SKH1 hairless mice), the chronically stressed group were found to be at higher risk of developing squamous cell carcinoma than the non-stressed 
group. The stressed group had a shorter latency prior to the development of the first tumour, lower INF- $\gamma$ expression, more infiltrating and circulating levels of regulatory $\mathrm{T}$ cells and reduced $\mathrm{T}$ helper cell infiltration (49). Therefore, chronic stress also impairs the immune system's ability to fight cancer by reducing anti-tumour lymphocyte and NK cell activity and increasing immunosuppression.

Gene modulation. In another murine study, chronic stimulation of the SNS during chronic stress was observed to interfere with the p53 tumour suppressor gene. The HPA axis activation was also thought to lead to elevated glucocorticoids with systemic increases in the expression of p53 inhibitors (namely MDM2). The effects of chronic stress on $\mathrm{p} 53$ reduces its normal critical tumour suppressor and anti-angiogenic roles (50). Chronic stress in murine prostate cancer models have also implicated catecholamines in promoting tumour growth by phosphorylating and inactivating the proapoptotic molecule Bcl-2-associated death promoter (51).

Angiogenesis and invasiveness. In an ovarian cancer model, chronic stress led to higher levels of tissue catecholamines, the downstream increased expression of angiogenic factors, (including vascular endothelial growth factor), and increased formation of pro-invasive enzymes (such as matrix metalloproteinase-2 and -9) by protein kinase A stimulation (51). Chronic stress may stimulate angiogenesis and increase invasiveness of some tumours, leading to increased tumour burden.

\section{Management}

Rationale. There is a requirement to effectively treat depression in cancer patients in order to improve QOL and survival $(4,5)$. It has been demonstrated that the treatment and improvement of depression in metastatic breast cancer, and improvement of depressive symptoms within the first year, significantly prolongs median survival time by 28.5 months compared with patients that experienced an increase in depressive symptoms (52). Both psychosocial interventions and pharmacotherapy are effective in treating depression in cancer, but the optimal combination and delivery of treatments are unknown and further research is required (53). The management of depression is likely to be different in each patient.

Medications. The two main classes of medication for depression in cancer are tricyclic antidepressants (TCAs) and selective serotonin reuptake inhibitors (SSRIs) (12). The exact mechanisms of action for these drugs has not been fully established, however, they are thought to cause adaptive changes in the brain and, over time, increase 5-HT neurotransmission leading to an improved mood $(26,54)$.

TCAs have more adverse effects and a higher risk of overdose compared with SSRIs. However, a meta-analysis found no recommendation for one antidepressant type over another in cancer due to a lack of research (55). The prescription of SSRIs must be carefully considered in patients receiving chemotherapy or radiotherapy, as SSRIs can often worsen emesis and nausea (53), whilst the anticholinergic effects of TCAs may worsen delirium associated with chemotherapy $(26,53)$.

Terminal patients. The use of conventional antidepressants in patients with terminal cancer may be unwise due to the delayed mode of action of these drugs $(12,53,54)$. An observational study supported this notion, concluding that currently prescribed antidepressants have little effect on improving depression in terminally ill depressed cancer patients, as measured by depression scores (56). In one trial, methylphenidate provided moderate to marked improvement in depression symptoms in $73 \%$ of depressed oncology patients within two days; this drug may therefore be an effective alternative to conventional antidepressants (57). However, whilst trials of methylphendidate have been promising, physiological tolerance quickly develops and doses must be increased $(57,58)$. Ketamine has recently been studied for its rapid and effective antidepressant effects, due to its antagonism of NMDA receptors (58). Ketamine has been suggested as a treatment of depression in terminally ill cancer patients where rapid reversal of depression is vital. A trial in a single patient with advanced cancer, however, exerted initially positive but unsustainable effects; larger randomised trials are necessary to assess the role of ketamine in treating depression in terminal patients (59).

Interactions. Chemotherapeutics may interact with antidepressants and cause nervous system toxicity, by reducing the metabolism of the antidepressants or by additive effects of the cancer drugs themselves. Some antidepressants may reduce the efficacy of chemotherapeutic drugs. A common example of this is the interaction of certain SSRIs (including fluoxetine and paroxetine, which are concurrently prescribed in $20-30 \%$ of breast cancer patients) with tamoxifen, which reduces the metabolism of tamoxifen to its active metabolite, endoxifen, by inhibiting the hepatic CYP2D6 enzyme. This reduces the effectiveness of the drug and increases the risk of breast cancer relapse $(60,61)$. Other SSRIs and herbal preparations containing hypercium (St John's Wort) may induce the CYP3A4 enzyme, which may lead to a reduction in levels of hepatically excreted anticancer agents, or an increase in the levels of drugs activated by CYP3A4 (62). Cancer drugs commonly have a narrow therapeutic range, therefore it is essential to avoid altering their levels. Great care must be taken in avoiding drug interactions (63).

Non-pharmacological. Positive patient-physician relationships and communication markedly reduces levels of distress in patients (15). In addition patients may benefit from a range of approaches, including relaxation strategies, psychoeducation, cognitive behavioural therapy (CBT), problem solving therapy (PST) and acceptance and commitment therapy (ACT) (64-68).

Relaxation strategies, including meditation and progressive muscle relaxation, enable patients to relieve mental and physical tension, thereby reducing stress, and have been observed to improve depression and QOL in cancer patients $(64,68)$. Psychoeducation may be used to build knowledge and coping strategies with regard to cancer, reducing uncertainty and anxiety. CBT identifies and equips patients with the skills to overcome maladaptive thought patterns and encourages emotional readjustment. Both psychoeducation and CBT have 
been demonstrated to be effective in improving depressive symptoms and QOL $(65,68)$. PST focuses on creating, implementing and appraising solutions for the manageable problems affecting patients, including relationships and financial problems. PST may improve psychological outcomes and QOL in depressed patients (66). ACT teaches patients how to tolerate difficult thoughts without being overwhelmed or dominated by them and develops psychological flexibility. ACT has been demonstrated to be equivalent to $\mathrm{CBT}$ in terms of its effect on mood and QOL (68).

Exercise therapy may also be effective in providing modest relief from depression in cancer patients. A meta-analysis concluded that, although there was a scarcity of research using depression as the primary endpoint, exercise may lower pain and improve fatigue and QOL among cancer survivors (69).

\section{Conclusion}

Depression remains an under-recognised comorbidity in cancer patients, with major implications on patient suffering, mortality and healthcare expenditure. Depression in cancer is markedly different from depression in healthy individuals, and involves a unique symptomatology and a strong biological aetiology. In the future, novel anti-inflammatory drugs may be able to provide more rapid and effective relief compared with current antidepressant therapy, by targeting the extensive inflammatory and endocrine pathways thought to underlie depression in cancer patients. At present, however, further research is required to identify the most effective combinations of pharmacological and psychosocial treatments for depression in cancer.

\section{References}

1. Mishel MH, Hostetter T, King B and Graham V: Predictors of psychosocial adjustment in patients newly diagnosed with gynecological cancer. Cancer Nurs 7: 291-299, 1984.

2. Linden W, Vodermaier A, Mackenzie R and Greig D: Anxiety and depression after cancer diagnosis: prevalence rates by cancer type, gender, and age. J Affect Disord 141: 343-351, 2012.

3. Brintzenhofe-Szoc KM, Levin TT, Li Y, Kissane DW and Zabora JR: Mixed anxiety/depression symptoms in a large cancer cohort: prevalence by cancer type. Psychosomatics 50: 383-391, 2009

4. Colleoni M, Mandala M, Peruzzotti G, et al: Depression and degree of acceptance of adjuvant cytotoxic drugs. Lancet 356 : 1326-1327, 2000.

5. Pinquart M and Duberstein PR: Depression and cancer mortality: a meta-analysis. Psychol Med 40: 1797-1810, 2010.

6. Satin JR, Linden W and Phillips MJ: Depression as a predictor of disease progression and mortality in cancer patients: a meta-analysis. Cancer 115: 5349-5361, 2009.

7. Lemon J, Edelman S and Kidman AD: Perceptions of the "Mind-Cancer" Relationship Among the Public, Cancer Patients, and Oncologists. J Psychosoc Oncol 21: 43-58, 2004.

8. American Psychiatric Association: Diagnostic and Statistical Manual of Mental Disorders. 5th edition. American Psychiatric Publishing, Arlington, VA, 2013.

9. Sneeuw KCA, Aaronson NK, van Wouwe MCC, et al: Prevalence and screening of psychiatric disorder in patients with early stage breast cancer. Qual Life Res 2: 50-51, 1993.

10. Okamura M, Yamawaki S, Akechi T, et al: Psychiatric disorders following first breast cancer recurrence: prevalence, associated factors and relationship to quality of life. Jpn J Clin Oncol 35: 302-309, 2005.

11. Ciaramella A and Poli P: Assessment of depression among cancer patients: the role of pain, cancer type and treatment Psychooncology 10: 156-165, 2001.
12. Ng CG, Boks MP, Zainal NZ and de Wit NJ: The prevalence and pharmacotherapy of depression in cancer patients. J Affect Disord 131: 1-7, 2011.

13. Allen R, Newman SP and Souhami RL: Anxiety and depression in adolescent cancer: findings in patients and parents at the time of diagnosis. Eur J Cancer 33: 1250-1255, 1997.

14. von Essen L,Enskär K, Kreuger A, et al: Self-esteem, depression and anxiety among Swedish children and adolescents on and off cancer treatment. Acta Paediatr 89: 229-236, 2000.

15. Boyes AW, Girgis A, Zucca AC and Lecathelinais C: Anxiety and depression among long-term survivors of cancer in Australia: results of a population-based survey. Med J Aust 190: S94-S98, 2009.

16. Spiegel D, Sands S and Koopman C: Pain and depression in patients with cancer. Cancer 74: 2570-2578, 1994.

17. Akechi T, Nakano T, Akizuki N, et al: Somatic symptoms for diagnosing major depression in cancer patients. Psychosomatics 44: 244-248, 2003.

18. Pasquini M, Speca A, Mastroeni S, et al: Differences in depressive thoughts between major depressive disorder, IFN-alpha-induced depression, and depressive disorders among cancer patients. J Psychosom Res 65: 153-156, 2008.

19. Musselman DL, Miller AH, Porter MR, et al: Higher than normal plasma interleukin- 6 concentrations in cancer patients with depression: preliminary findings. Am J Psychiatry 158: 1252-1257, 2001.

20. Nadjar A, Bluthé RM, May MJ, et al: Inactivation of the cerebral NFkappaB pathway inhibits interleukin-1beta-induced sickness behavior and c-Fos expression in various brain nuclei. Neuropsychopharmacology 30: 1492-1499, 2005.

21. Zigmond AS and Snaith RP: The hospital anxiety and depression scale. Acta Psychiatr Scand 67: 361-370, 1983.

22. Jehn CF, Kuehnhardt D, Bartholomae A, et al: Biomarkers of depression in cancer patients. Cancer 107: 2723-2729, 2006.

23. Bianchi ME: DAMPs, PAMPs and alarmins: all we need to know about danger. J Leukoc Biol 81: 1-5, 2007.

24. Aggarwal BB, Shishodia S and Sandur SK: Inflammation and cancer: how hot is the link? Biochem Pharmacol 72: 1605-1621, 2006.

25. Bierhaus A, Wolf J, Andrassy M, et al: A mechanism converting psychosocial stress into mononuclear cell activation. Proc Natl Acad Sci USA 100: 1920-1925, 2003.

26. Szabo S, Gould TD and Manji HK: Introduction to neurotransmitters, receptors, signal transduction, and second messengers in psychiatric disorders. In: The American Psychiatric Publishing Textbook of Psychopharmacology. Schatzberg AF and Nemeroff CB (eds). 3rd edition. American Psychiatric Publishing, Washington DC, pp3-51, 2004.

27. Raison CL, Capuron L and Miller AH: Cytokines sing the blues: inflammation and the pathogenesis of depression. Trends Immunol 27: 24-31, 2006.

28. Zhu CB, Carneiro AM, Dostmann WR, Hewlett WA and Blakely RD: p38 MAPK activation elevates serotonin transport activity via a trafficking-independent, protein phosphatase 2A-dependent process. J Biol Chem 280: 15649-15658, 2005.

29. Holsboer F and Ising M. Central CRH system in depression and anxiety - evidence from clinical studies with CRH1 receptor antagonists. Eur J Pharmacol 583: 350-357, 2008.

30. Duman RS and Monteggia LM: A neurotrophic model for stress-related mood disorders. Biol Psychiatry 59: 1116-1127, 2006

31. Felger JC, Mun J, Kimmel HL, Nye JA, et al: Chronic interferon- $\alpha$ decreases dopamine 2 receptor binding and striatal dopamine release in association with anhedonia-like behaviour in nonhuman primates. Neuropsychopharmacology 38: 2179-2187, 2013.

32. Felger JC, Li L, Marvar PJ, et al: Tyrosine metabolism during interferon-alpha administration: association with fatigue and CSF dopamine concentrations. Brain Behav Immun 31: 153-160, 2013.

33. Dantzer R, O'Connor JC, Freund GG, et al: From inflammation to sickness and depression: when the immune system subjugates the brain. Nat Rev Neurosci 9: 46-56, 2008.

34. DeMyer MK, Shea PA, Hendrie HC and Yoshimura NN: Plasma tryptophan and five other amino acids in depressed and normal subjects. Arch Gen Psychiatry 38: 642-646, 1981.

35. Myint AM and Kim YK: Cytokine-serotonin interaction through IDO: a neurodegeneration hypothesis of depression. Med Hypotheses 61: 519-525, 2003.

36. Sapolsky RM: The possibility of neurotoxicity in the hippocampus in major depression: a primer on neuron death. Biol Psychiatry 48: 755-765, 2000. 
37. Pace TW, Hu F and Miller AH: Cytokine-effects on glucocorticoid receptor function: relevance to glucocorticoid resistance and the pathophysiology and treatment of major depression. Brain Behav Immun 21: 9-19, 2007.

38. Pavlov VA and Tracey KJ: The cholinergic anti-inflammatory pathway. Brain Behav Immun 19: 493-499, 2005.

39. Besedovsky HO and del Rey A: Immune-neuro-endocrine interactions: facts and hypotheses. Endocr Rev 17: 64-102, 1996.

40. Wang X: Interleukin 1alpha (IL-1alpha) induced activation of p38 mitogen-activated kinase inhibits glucocorticoid receptor function. Mol Psychiatry 9: 65-75, 2004.

41. Raison CL and Miller AH: When not enough is too much: the role of insufficient glucocorticoid signaling in the pathophysiology of stress-related disorders. Am J Psychiatry 160: 1554-1565, 2003.

42. Patten SB and Barbui C. Drug-induced depression: a systematic review to inform clinical practice. Psychother Psychosom 73 207-215, 2004.

43. Fann JR, Thomas-Rich AM, Katon WJ, et al: Major depression after breast cancer: a review of epidemiology and treatment. Gen Hosp Psychiatry 30: 112-126, 2008.

44. Liu L, Fiorentino L, Natarajan L, et al: Pre-treatment symptom cluster in breast cancer patients is associated with worse sleep, fatigue and depression during chemotherapy. Psychooncology 18 187-194 2009.

45. Koenig HG, Shelp F, Goli V, et al: Survival and health care utilization in elderly medical inpatients with major depression. J Am Geriatr Soc 37: 599-606, 1989.

46. Misono S, Weiss NS, Fann JR, et al: Incidence of suicide in persons with cancer. J Clin Oncol 26: 4731-4738, 2008

47. Michael Maes M, Meltzer HY, Stevens W, et al: Natural killer cell activity in major depression: relation to circulating natural killer cells, cellular indices of the immune response, and depressive phenomenology. Prog Neuropsychopharmacol Biol Psychiatry 18: 717-730, 1994.

48. Lutgendorf SK, Lamkin DM, DeGeest K, et al: Depressed and anxious mood and T- cell cytokine expressing populations in ovarian cancer patients. Brain Behav Immun 22: 890-900, 2008

49. Saul AN, Oberyszyn TM, Daugherty C, et al: Chronic stress and susceptibility to skin cancer. J Natl Cancer Inst 97: 1760-1767, 2005.

50. Feng Z, Liu L, Zhang C, et al: Chronic restraint stress attenuates p53 function and promotes tumorigenesis. Proc Natl Acad Sci USA 109: 7013-7018, 2012.

51. Hassan S, Karpova Y, Baiz D, et al: Behavioral stress accelerates prostate cancer development in mice. J Clin Invest 123: 874-886, 2013.

52. Giese-Davis J, Collie K, Rancourt KM, et al: Decrease in depression symptoms is associated with longer survival in patient with metastatic breast cancer: a secondary analysis. J Clin Oncol 29: 413-420, 2011

53. Li M,Fitzgerald P, Rodin G: Evidence-based treatment of depression in patients with cancer. J Clin Oncol 30: 1187-1196, 2012.

54. Norman TR: The new antidepressants - mechanisms of action. Aust Prescr 22: 106-108, 1999.
55. Laoutidis ZG, Mathiak K: Antidepressants in the treatment of depression/depressive symptoms in cancer patients: a systematic review and meta-analysis. BMC Psychiatry 13: 140, 2013.

56. Lloyd-Williams M, Payne S, Reeve J and Kolamunnage Dona R: Antidepressant medication in patients with advanced cancer - an observational study. QJM 106: 995-1001, 2013.

57. Olin J and Masand P: Psychostimulants for depression in hospitalized cancer patients. Psychosomatics 37: 57-62, 1996.

58. Stefanczyk-Sapieha L, Oneschuk D, Demas M: Intravenous ketamine "burst" for refractory depression in a patient with advanced cancer. J Palliat Med 11: 1268-1271, 2008.

59. Berman RM, Cappiello A, Anand A, et al. Antidepressant effects of ketamine in depressed patients. Biol Psychiatry 47: 351-354, 2001.

60. Caraci F, Crupi R, Drago F and Spina E: Metabolic drug interactions between antidepressants and anticancer drugs: focus on selective serotonin reuptake inhibitors and hypericum extract. Curr Drug Metab 12: 570-577, 2011.

61. Borges S, Desta Z, Li L, et al: Quantitative effect of CYP2D6 genotype and inhibitors on tamoxifen metabolism: implications for optimization of breast cancer treatment. Clin Pharmacol Ther 80: 61-74, 2006.

62. Whitten DL, Myers SP, Hawrelak JA and Wohlmuth H: The effect of St John's wort extracts on CYP3A: a systematic review of prospective clinical trials. Br J Clin Pharmacol 62: 512-526, 2006.

63. Yap KY, Tay WL, Chui WK and Chan A: Clinically relevant drug interactions between anticancer drugs and psychotropic agents. Eur J Cancer Care (Engl) 20: 6-32, 2011.

64. Sloman R: Relaxation and imagery for anxiety and depression control in community patients with advanced cancer: Cancer Nurs 25: 432-435, 2002.

65. Osborn RL, Demoncada AC and Feuerstein M: Psychosocial interventions for depression, anxiety, and quality of life in cancer survivors: meta-analyses. Int J Psychiatry Med 36: 13-34, 2006.

66. Hopko DR, Armento ME, Robertson SM, et al: Brief behavioural activation and problem-solving therapy for depressed breast cancer patients: randomized trial. J Consult Clin Psychol 79: 834-849, 2011.

67. Feroes DL, Lane L, Ciarrochi J and Blackledge JT: Acceptance and Commitment Therapy (ACT) for improving the lives of cancer patients: a preliminary study. Psychooncology 22: 459-464, 2013.

68. Barsevick AM, Sweeney C, Haney E and Chung E. A systematic qualitative analysis of psychoeducational interventions for depression in patients with cancer: Oncol Nurs Forum 29: 73-87, 2002.

69. Craft LL, Vanlterson EH, Helenowski IB, et al: Exercise effects on depressive symptoms in cancer survivors: a systematic review and meta-analysis. Cancer Epidemiol Biomarkers Prev 21: $3-19,2012$. 